\title{
A Queueing Analysis of a Security-Check System with Two Types of Inspection Channels
}

\author{
Chia-Hung Wang \\ College of Information Science and Engineering, Fujian University of Technology, Fuzhou City, Fujian Province 350118, China \\ Fujian Provincial Key Laboratory of Big Data Mining and Applications, Fuzhou City, Fujian Province 350118, China
}

\begin{abstract}
This paper studies a security-check system at the high-speed railway stations with two types of inspection channels, including Normal channels and Green channels. Here, general passengers go through the strict inspections in the Normal channels with unlimited waiting space, whereas special passengers are offered a fast inspection in the Green channels with a finite capacity. To investigate the stationary behavior of the studied security-check system, a queueing model is developed through deriving the two-dimensional quasi-birth-and-death (QBD) process. In addition, a matrix-geometric solution is presented in the paper to determine the steady-state probability and evaluate the system performance of interest, such as the average queue length and average waiting time. A sensitivity analysis is also conducted in the numerical experiments to demonstrate the managerial impact of varying the finite capacity in Green channels on the overall system performance.
\end{abstract}

Keyword-security check; queueing system; green channels; finite capacity; matrix geometric method

\section{INTRODUCTION}

In China, the high-speed railway has been extended to 29 provincial-level administrative divisions, and it has been passenger-dedicated with over $25,000 \mathrm{~km}$ in total length. In 2017, it was reported that 1.713 billion passengers were delivered through Chinese high-speed railway, and the total cumulative number of trips reached to 7 billion. With the continuous development of high-speed railway in China, the railway stations definitely play a more and more important role in the transportation of passengers.

The top legislature in China, The National People's Congress of the People's Republic of China, passed the country's first anti-terrorism law at the end of the 18th meeting of the 12th National People's Congress Standing Committee [1] It stipulates that the management units should conduct security checks on the passengers, personal belongings and vehicles that enter the (high-speed) railway stations according to the AntiTerrorism Act of the People's Republic of China, implemented from January 1, 2016. It not only provides legal support and guarantees for the nation to combat terrorist activities, safeguard national and public security, but also offers the security of lives and property, and strengthens the antiterrorism cooperation in the international community. Besides, it is an important policy to ensure safety and security through strict security-check procedures at the high-speed railway stations in China. Therefore, the security-check system has become vital for the safety of high-speed railway transportation.
Under the environment of large passenger flow, the management units of high-speed railway stations conduct strict inspections on inbound passengers and their personal belongings at the same time. The serious contradiction between the strict security standards and the operational efficiency/convenience makes the security-check procedures more challenging in the stations [2], [3]. On the basis of strengthening the function of supervision and improving the efficiency and quality of inspection, the management units continuously seek opportunities to reduce the security-check costs without negatively affecting the satisfaction level of passengers. Thus, there is an urgent need to investigate and explore the efficient security-check systems.

Nowadays, the growing terrorist threats make the study of security-check systems become a hot research issue, such as [4] [5], [6], [7], [8] and reference therein. By offering some passengers a time window during which they could arrive to enter a priority queue, the possibilities of implementing virtual queuing at airport security lanes were investigated in [9]. In [10], two main conflicting goals (high security level versus good customer service) were analyzed for a border-crossing security-check station. Using a stylized two-stage queueing model, a security-check system between the U.S.-Canadian border crossings was investigated in [11] with both security and customer service goals. Besides, a multi-server queuing model was developed in [12] to estimate the delay in support of a predictive traveler information system for the border-crossing passengers.

In order to study how to effectively utilize the selectee lane resource, a simulation-based queueing design framework was discussed in [13] for screening passengers. Through a steadystate analysis of multi-server queueing model, the system congestion and risk level of a risk-based checkpoint screening system with two types of inspection queues were explored in [14]. For the freight inspections, the serious congestion problems and bottlenecks within the security checkpoints were discussed in [15], where it was explained that the inspection cost and delays would cause a huge loss of product value in the supply chain. Furthermore, according to [16], the marginal increase in security per dollar spent is significantly lower when non-selectee checked bags are screened than when only selectee checked bags are screened.

Compared with the relevant research works mentioned above, this paper studies a security-check system with two types of inspection channels, including Green channels and Normal channels. Through the Green channels, it can help 
special passengers quickly go through a security-check procedure, such as VIP, government officials, and soldiers on duty, etc. On the other hand, the general passengers receive the strict security-check procedure and inspections in the Normal channels. In this work, we not only derive a queueing model to understand the steady-state behavior of the studied system, but also present a matrix-geometric recursive algorithm to evaluate the influence of varying the finite capacity in Green channels on the overall system performance.

The main contribution of this paper is to provide an analytic mechanism for managing a security-check system with two types of inspection channels. The steady-state analysis of the studied security-check system will be derived through a twodimensional state dependent quasi-birth-and-death (QBD) queueing model. By using a matrix geometric method, the stationary probability and the system performance of our interest will be determined for the proposed queueing model. Moreover, the sensitivity analysis presented in our numerical results could contribute managerial insights for optimizing the finite capacity in the studied security-check system at the highspeed railway stations.

The remainder of this paper is organized as follows. In Section 2, we introduce a security-check system with two types of inspection queues. In Section 3, a queueing analysis of the studied system is derived through a two-dimensional state dependent quasi-birth-and-death queueing model. In Section 4, a sensitivity analysis is presented to investigate the managerial effect of varying the finite capacity in Green channels on the system performance of our interest. Finally, the concluding remarks are summarized in Section 5.

\section{A SECURITY-CHECK SYSTEM WITH TwO TYPES OF INSPECTION QUEUES}

As shown in Figure 1, there are two types of inspection queues in the security-check system: Normal channels with the strict scrutiny and Green channels with a quick inspection. The management units of high-speed railway stations execute strict inspections for normal passengers in Normal channels with unlimited waiting space, whereas they execute a fast inspection in Green channels with a limited capacity. When the current queue length in Green channels reaches its capacity limit, all incoming passengers will be directed to the Normal channels for the strict security check.

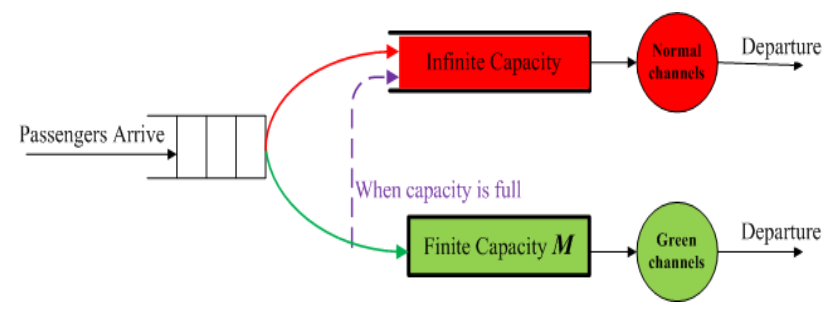

FIGURE I. A SECURITY-CHECK SYSTEM WITH TWO TYPES OF INSPECTION CHANNELS.

When passengers arrive at the security checkpoints, they are differentiated into two different risk classes based on their identities and attributes, and then go through the appropriate type of inspection channels, where a security-check procedure and screening devices are used to identify a (potential) threat. In each type of inspection queue, passengers are screened based on a First-Come-First-Served order.

The normal security-check procedure consists of two operations: inspecting the passengers and inspecting their carry-on bags. The total time required for inspecting a passenger is affected by not only the procedure required for his/her identity check, but also the throughput rate of the electronic equipments and a manual search. The system congestion of the studied security-check system will be determined through a queueing analysis of two-dimensional state dependent QBD process.

\section{A QUEUEING ANALYSis}

In this section, we develop a queueing model to understand the stationary behavior of the system, and present a matrix geometric method to determine the steady-state probability. In addition, we present a matrix-geometric recursive algorithm to evaluate the impact of the finite capacity in Green channels on the performance measures.

We consider a queueing model for screening passengers at the security checkpoints with two type of inspection channels (Green channels and Normal channels), as shown in Figure 1. Suppose the arrival of incoming passengers at the security checkpoints follows a Poisson process with mean rate $\lambda$. It offers guaranteed delay time in Green channels with a limited waiting space, denoted by $M$. Passengers waiting in each type of inspection channels are screened in First-Come-First-Served order. Assume that the screening time of passengers follow exponential distributions with the average service rate $\mu_{1}$ in Normal channels and the average service rate $\mu_{2}$ in Green channels, respectively.

Let $X_{1}(t)$ and $X_{2}(t)$ be the current queue lengths (including those passengers in inspection service) in Normal channels and Green channels, respectively. The system state can be defined as $\left(X_{1}(t), X_{2}(t)\right)$ on the state space $\boldsymbol{S}=\{(i, j) \mid i=0,1,2, \ldots$; $j=0,1,2, \ldots, M\}$. At the system state $(i, j)$, we suppose that an incoming passenger would be assigned to Normal channels with a state-dependent arrival rate $\lambda_{1}(i, j)$, and there is a statedependent arrival rate $\lambda_{2}(i, j)$ to Green channels. It is trivial that $\lambda_{1}(i, j)+\lambda_{2}(i, j)=\lambda$ for all system states $(i, j)$ belong to the set $\boldsymbol{S}$.

The system states of the studied queueing model can be classified into three categories: the states in category I are "all join Normal channels" states; the states in category II are "join Normal channels or Green channels" states; and the states in category III are "all join Green channels". The transition rate from current state $(i, j)$ to the next state $\left(i, j^{\prime}\right)$ is given by the elements of the infinitesimal generator matrix $\mathbf{\Omega}$. This queueing model can be formulated as a state dependent QBD process, where the state transition depends on the current queueing states of the system.

Based on the classification of the system states, we can construct the infinitesimal generator matrix $\boldsymbol{\Omega}$ as follows: 


$$
\mathbf{\Omega}=\left[\begin{array}{cccccccccc}
\mathbf{B}_{0,0} & \mathbf{C}_{0,1} & \mathbf{0} & & \ldots & & & & & \\
\mathbf{A} & \mathbf{B}_{1,1} & \mathbf{C}_{1,2} & & \mathbf{0} & \ldots & & & & \\
\mathbf{0} & \mathbf{A} & \mathbf{B}_{2,2} & & \mathbf{C}_{2,3} & \mathbf{0} & \ldots & & & \\
\vdots & \mathbf{0} & \ddots & \ddots & \ddots & & \ddots & & & \\
\vdots & \vdots & \ddots & \mathbf{A} & \mathbf{B}_{M, M} & \mathbf{C}_{M, M+1} & \mathbf{0} & \\
\mathbf{0} & \vdots & & \mathbf{0} & \mathbf{A} & & \mathbf{B} & \mathbf{C} & \mathbf{0} \\
\vdots & & & \mathbf{0} & & \ddots & & \ddots & \ddots & \ddots
\end{array}\right]
$$

where the dimensions of all submatrices are $(M+1)$ by $(M+1)$.

Under the stability condition, the stationary probability vector is defined as $\boldsymbol{\pi}_{n}=\left(\boldsymbol{\pi}_{n, 0}, \boldsymbol{\pi}_{n, 1}, \boldsymbol{\pi}_{n, 2}, \ldots, \boldsymbol{\pi}_{n, M}\right)$, where indices $n=0,1,2, \ldots$, denote the queue length of Normal channels in steady states. When $n \geqq M$, the matrix-geometric solution for such a QBD process can be obtained by $\boldsymbol{\pi}_{n+1}=\boldsymbol{\pi}_{n} \cdot \mathbf{R}$, where $\mathbf{R}$ is the rate matrix satisfying $\mathbf{R}^{2} \cdot \mathbf{A}+\mathbf{R} \cdot \mathbf{B}+\mathbf{C}=\mathbf{0}$. For $0 \leq n \leq M$, the probability vector $\boldsymbol{\pi}_{n}$ can be obtained by solving a set of equations consisting of the boundary conditions $\boldsymbol{\pi} \cdot \boldsymbol{\Omega}=\mathbf{0}$ and the normalization condition. Note that, in the case of large-scale size $M$, it would result in a large number of boundary states and phases of the QBD process, which would greatly increase the computational complexity. For further discussions on efficiently solving the matrix-geometric solution for a QBD process, interested readers may refer to [17] and [18].

To efficiently compute the stationary probabilities, we present a matrix-geometric recursive algorithm as follows:

Step 1. Compute a matrix $\boldsymbol{\Psi}=-[\mathbf{B}+\boldsymbol{\Phi}] \cdot \mu_{1}^{-1}$, where we define a $(M+1)$ by $(M+1)$ matrix $\boldsymbol{\Phi}=\left[\begin{array}{ccc}0 & \cdots & 0 \mu_{1} \\ \vdots & \ddots & \vdots \\ 0 & \cdots & 0 \mu_{1}\end{array}\right]$.

Step 2. For matrix $\boldsymbol{\Psi}$, we determine an eigenvalue $\sigma$ which lies between zero and one, and then obtain its corresponding normalized right eigenvector $\boldsymbol{v}=\left(v_{1}, v_{2}, \cdots, v_{M+1}\right)$.

Step 3. Calculate a rate matrix $\mathbf{R}=h\left[\begin{array}{c}0 \\ \boldsymbol{v}^{\mathrm{T}}\end{array}\right]$, where we take $h=\frac{\sigma}{v_{M+1}}$, for later use in Step 6 and iterations in Step 7.

Step 4. Construct a sequence of matrices $\mathbf{T}_{0}, \mathbf{T}_{1}, \ldots, \mathbf{T}_{M}$, where we set $\mathbf{T}_{0}=\mathbf{I}, \quad \mathbf{T}_{1}=-\mathbf{B}_{0,0} \cdot \mathbf{A}^{-1}$, and $\mathbf{T}_{i}=$ $-\left(\mathbf{T}_{i-2} \cdot \mathbf{C}_{i-2, i-1}+\mathbf{T}_{i-1} \cdot \mathbf{B}_{i-1, i-1}\right) \cdot \mathbf{A}^{-1}$ for all $2 \leq i \leq M$.

Step 5. Set up an initial solution $\boldsymbol{\pi}_{i}=\boldsymbol{\pi}_{0} \cdot \mathbf{T}_{i}$, for all $0 \leq$ $i \leq M$.

Step 6 . Calculate the steady-state probability $\boldsymbol{\pi}_{0}$ by solving the following simultaneous equations:

$$
\begin{gathered}
\boldsymbol{\pi}_{0} \cdot\left(\mathbf{T}_{M-1} \cdot \mathbf{C}_{M-1, M}+\mathbf{T}_{M} \cdot(\mathbf{B}+\mathbf{R} \cdot \mathbf{A})\right)=\mathbf{0}, \\
\boldsymbol{\pi}_{0} \cdot\left[\sum_{i=0}^{M-1} \mathbf{T}_{i} \cdot \mathbf{1}+\mathbf{T}_{M} \cdot\left[\begin{array}{c}
1 \\
\vdots \\
1 / \square
\end{array}\right]\right]=1 .
\end{gathered}
$$

Step 7. Iteratively determine the remainder steady-state probabilities $\pi_{M+j}, \forall j \geq 1$ through the recurrence relation $\boldsymbol{\pi}_{M+j}=\boldsymbol{\pi}_{M} \cdot \sigma^{j-1} \cdot \mathbf{R}$.

After determining the stationary probabilities, we can evaluate the system performance of our interest, including the average queue length and average waiting time.

\section{NUMERICAL RESULTS}

In this section, we conduct a sensitivity analysis to study the managerial effect of the finite capacity $M$ on the system performance. Our numerical experiments are conducted with MATLAB on the PC platform with Intel ${ }^{\circledR}$ Core $^{\mathrm{TM}}$ i7-3770 CPU@3.40 GHz and 32 GB RAM.

In the numerical experiments, the parameters in the proposed queueing model are set as follows. For the case of heavy traffic load, we have the average arrive rate $\lambda=91$ passengers per minute; on the other hand, we take the average arrive rate $\lambda=76$ passengers per minute for the light traffic case. The average service rate of security checkpoints in Normal channels is $\mu_{1}=45$ passengers per minute, and the average service rate of security checkpoints in Green channels is $\mu_{2}=65$ passengers per minute. The finite capacity size of Green channels, $M$, varies from 3 to 20 . Under these two traffic cases (the heavy traffic load and the light traffic load), the numerical results of the average queue length and average waiting time are depicted in Figure 2 and Figure 3, individually. The red line indicates the case of heavy traffic load, and the black line represents the case of light traffic load.

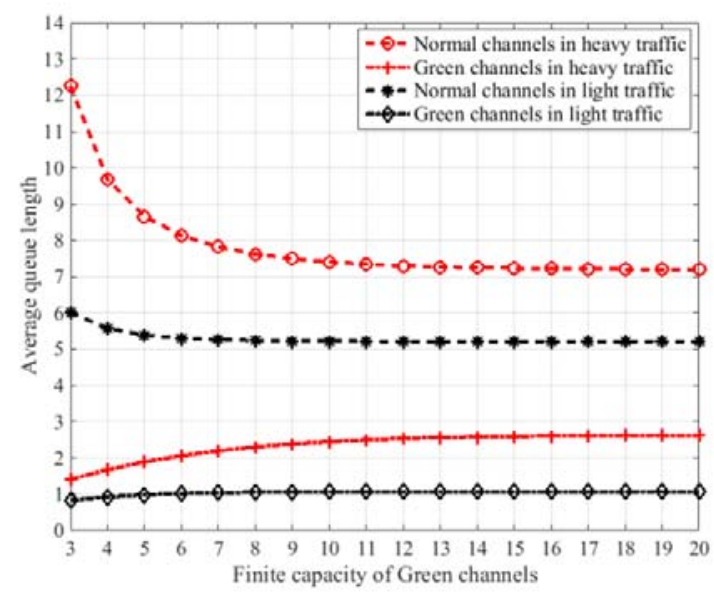

FIGURE II. THE AVERAGE QUEUE LENGTH VERSUS THE FINITE CAPACITY IN GREEN CHANNELS.

Figure 2 illustrates the average queue lengths of passengers in both the Normal channels and the Green channels at the same time. We find that the average queue length of passengers in the Green channels will be increased when enlarging its finite capacity $M$, and it approaches to a stable mean value when the finite capacity is enough for a given traffic load. This is because a certain proportion of special passengers may be directed to the Normal channels due to the insufficient capacity in Green channels. In addition, it is also observed that the average queue length for the Normal channels can be decreased 
while we increase the finite capacity $M$. Nevertheless, the improvement continuously decreases when enlarging the capacity, and it would approach to zero if a specific capacity is enough for serving special passengers in Green channels.

In Figure 3, it shows the effect of increasing the finite capacity $M$ on the average waiting time for both the heavy traffic load and the light traffic load. For each type of inspection channels, it can be found that the average waiting in the heavy traffic is always larger than the average waiting in the light traffic. Besides, we observe that the average waiting time of passengers in the Normal channels can be decreased when we enlarge the finite capacity $M$. However, the marginal improvement would decrease to zero if the finite capacity in Green channels is large enough for special passengers.

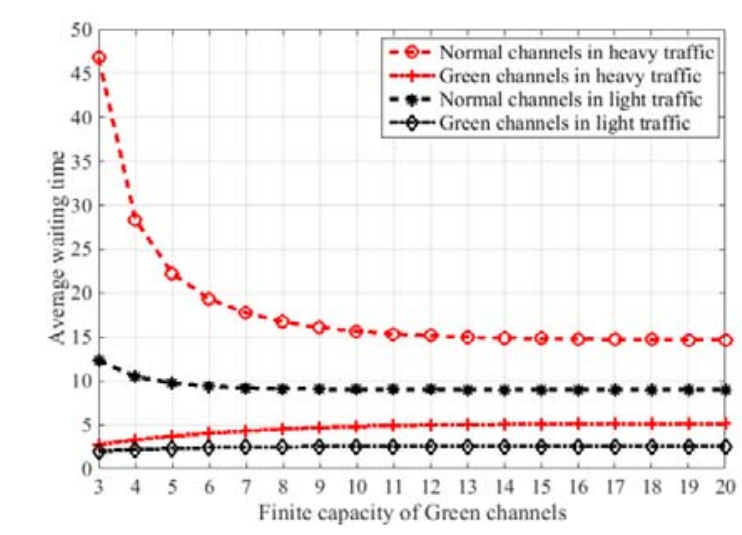

FIGURE III. THE AVERAGE WAITING TIME VERSUS THE FINITE CAPACITY IN GREEN CHANNELS.

\section{CONCLUSIONS}

In this paper, we investigate a security-check system with two types of inspection channels, where special passengers can be provided a fast inspection in the Green channels with limited waiting capacity, whereas general passengers are offered the strict inspection in the Normal channels with unlimited waiting space. To derive the steady-state analysis of the studied security-check system, a queueing model with two-dimensional state space was developed in our study through a matrix geometric method. Moreover, an efficient solution algorithm was presented to compute the stationary probability and system performance of the proposed queueing model. In the numerical results, the average queue length and the average waiting time were also determined under the heavy and light traffic cases.

In the numerical experiments, we observed the influence of varying finite capacity size of Green channels on the system performance. For each capacity size, we obtained both the average queue length and average waiting time for the Green channels and the Normal channels simultaneously. Besides, a sensitivity analysis was also presented in the paper while varying the finite capacity in two scenarios of heavy traffic load and light traffic load. From our numerical results, it illustrates that there are several significant trends in the change of the average waiting time and the average queue length in Normal channels and Green channels, individually.
Furthermore, we draw a conclusion that a proper control on the finite capacity in Green channels could balance the queueing efficiency of the security-check system and the capacity cost of serving special passengers.

\section{ACKNOWLEDGMENT}

This research was supported in part by Fujian Provincial Department of Science and Technology, China, under Grant No. 2016J01330, and the Education Department of Fujian Province, China, under Grant No. JAT160337.

\section{REFERENCES}

[1] The National People's Congress of the People's Republic of China, "The Anti-Terrorism Act of People's Republic of China," December 27, 2015. Available at http://www.npc.gov.cn/npc/xinwen/201512/28/content_1957401.htm

[2] E. Matsika, C. O'Neill, U. Battista, M. Khosravi, A. de Santiago Laporte, and E. Munoz, "Development of risk assessment specifications for analysing terrorist attacks vulnerability on metro and light rail systems," Transportation Research Procedia, vol. 14, pp. 1345-1354, 2016.

[3] C.-H. Wang, "A review of operational mechanisms and simulations for security screening systems," Computer Science and Application, vol. 7, no. 11, pp. 1067-1078, 2017.

[4] C.-H. Wang, H.P. Luh, and Z.G. Zhang, "Secure and efficient global supply chain management: a queueing analysis," Lecture Notes in Operations Research and Decision Science, pp. 28-36, 2012.

[5] C.-H. Wang, M.-E. Wu, and C.-M. Chen, "Inspection risk and delay for screening cargo containers at security checkpoints," Proc. 7th Int. Conf. on Intelligent Information Hiding and Multimedia Signal Processing (IIH-MSP-2015), Adelaide, Australia, IEEE Press, pp. 211-214, 2015.

[6] P. Huang, H.P. Luh, and Z.G. Zhang, "A queueing model for tiered inspection lines in airports," International Journal of Information and Management Sciences, vol. 27, pp. 147-177, 2016.

[7] C.-H. Wang, "Arena simulation for aviation passenger security-check systems," Advances in Intelligent Systems and Computing, vol. 536, pp. 95-102, 2016.

[8] C.-H. Wang and J. Lan, "Performance evaluation of a risk-based threetier inspection system," Proc. 2nd Int. Conf. on Computational Modeling, Simul. Applied Math. (CMSAM2017), Beijing, China, DEStech Transactions on Computer Science and Engineering, pp. 464-468, 2017.

[9] R. de Lange, I. Samoilovich, and B. van der Rhee, "Virtual queuing at airport security lanes," European Journal of Operational Research, vol. 225, pp. 153-165, 2013.

[10] Z.G. Zhang, "Performance analysis of a queue with congestion-based staffing policy," Management Science, vol. 55, pp. 240-251, 2009.

[11] Z.G. Zhang, H.P. Luh, and C.-H. Wang, "Modeling security check queues," Management Science, vol. 57, pp. 1979-1995, 2011.

[12] L. Lin, Q. Wang, and A.W. Sadek, "Border crossing delay prediction using transient multi-server queueing models," Transportation Research Part A, vol. 64, pp. 65-91, 2014.

[13] X. Nie, G. Parab, R. Batta, and L. Lin, "Simulation-based selectee lane queuing design for passenger checkpoint screening," European Journal of Operational Research, vol. 219, pp. 146-155, 2012.

[14] C.-H. Wang, "A modelling framework for managing risk-based checkpoint screening systems with two-type inspection queues," Proc. 3rd Int. Conf. on Robot, Vision and Signal Processing (RVSP 2015), Kaohsiung, Taiwan, IEEE Press, pp. 220-223, 2015.

[15] J.J. Ruiz-Aguilar, I.J. Turias, and M.J. Jiménez-Come, “A two-stage procedure for forecasting freight inspections at border inspection posts using SOMs and support vector regression," International Journal of Production Research, vol. 53, no. 7, pp. 2119-2130, 2015.

[16] J.L. Virta, S.H. Jacobson, and J.E. Kobza, "Analyzing the cost of screening selectee and non-selectee baggage," Risk Analysis, vol. 23, no. 5 , pp. 897-908, 2003. 
[17] M.F. Neuts, Matrix-Geometric Solutions in Stochastic Models. The John Hopkins University Press, 1981.

[18] H.P. Luh, Z.G. Zhang, and C.-H. Wang, "A computing approach to two competing services with a finite buffer effect," Proc. 8th Int. Conf. on Queueing Theory and Network Applications (QTNA2013), Taichung, Taiwan, pp. 15-21, 2013. 